\title{
Printed piezoresistive strain sensors for monitoring of light-weight structures
}

\author{
Rausch, Jacqueline \\ TU Darmstadt, Institut EMK \\ Merckstr. 25,64283 Darmstadt
}

\begin{abstract}
In this paper we present the design and test of printed strain sensors, which can be integrated in lightweight structures for monitoring purposes. We focus on composite structures consisting of a metal substrate and insulating and conductive ink layers for sensing normal strain at the surface. Both, inkjet and screen printing technology are used to realize resistive topologies. In a first step, we analyze electrical properties of functional inks: electrical impedance and breakdown electrical field strength in case of insulation inks, resistance in case of conducting inks. Silver and PEDOT:PSS based suspensions are printed as sensing layer. To determine the resistance change due to plastic deformation of the metal substrate, tensile tests are performed up to $30 \%$ strain and subsequent resistance change is measured. In a second step, the sensing effect of printed conductive structures is investigated. Resistive sensing topologies are designed for detecting longitudinal and transversal normal strain. Meander structures, which form single resistors as well as bridge configurations, are printed on test specimens and analyzed in a four-point bending set up. Performing loading and unloading cycles, gauge factor, cross sensitivity, nonlinearity and hysteresis error of the sensors are measured.
\end{abstract}

\section{Introduction}

The main idea of smart or intelligent structures is to functionalize the structure itself, so that it is able to detect and adapt to variable effects of the environment. For this purpose sensor-actuator systems are integrated into the structure, mechanical values like stress, strain, acceleration or force are measured and the electrical output signal is fed back to the signal processing unit to control the actuators. Within the German research programme LOEWE AdRIA (Adaptronic - Research, Innovation, Application) engineering, computer- and material scientists are investigating methods and components for solving vibration related problems with the objective of increasing usability and reducing manufacturing costs. One of the subprojects deals with cost-effective sensor and actuator integration. On this, rapid prototyping manufacturing techniques and novel materials are used to combine function and structural element. In this paper we present the design, realization and test of printed strain sensors, which can be integrated in light-weight structures for monitoring purposes. We focus on composite structures consisting of metal substrate as well as insulating and conductive ink layers for sensing normal strain at surfaces of measuring objects. One goal is to print sensors and actuators on substrates before a subsequent forming process. Both, inkjet and screen printing technologies are used to realize resistive topologies. The organization of the paper is as follows: The first chapter presents the state of science and technology of printed strain sensors. Functional inks and common printing methods are mentioned. The second chapter deals with the design and manufacturing of printed sensors on conducting substrates. The characterization of the printed sensors is described in the third part followed by a discussion and it closes with an outlook.

\section{State of Science and Technology}

Since the early 1970 s printing of resistive sensors is a common technique. Soldering pastes based on glass-ceramic composits are screen printed and thus sintered on glass, ceramic or metal substrates [1]. Nowadays water- or polymer-based suspensions with conducting particles are available, which can be printed on arbitrary surfaces. Submicron sized particles e.g. made of silver, gold, carbon or conducting polymers like PEDOT:PSS are comon $[3,4,5]$. Beside contacting purposes of microsystems, the printing of chemical and mechanical sensors is used to functionalize the surfaces. Mainly, insulating substrates like polymer foils are used as substrates [4-9]. To deposit thin sensing layers, contactless printing methods like aerosoljet and inkjet, but also coating in combination with photolithography is common. In the scientifical area novel conducting polymers like composites of polymer matrices and conducting nanoparticles are formulated [5, 7, 14]. Up to now, only the piezoresistive properties are analyzed under laboratory conditions. Table 1 summarizes important parameters of the inks, which have been used for printed strain sensors and a selection of the mentioned composites. 
Tab. 1 Comparison of important parameters of functional inks, which were used for strain sensors.

\begin{tabular}{c|c|c|c} 
Functional ink & Gauge factor & $\boldsymbol{\rho}$ in $\Omega \times \mathbf{m m}$ & Technology \\
\hline PEDOT:PSS & $0.48 \pm 0.07[11]$ & $2.51[11]$ & Coating [11, 13] \\
& $17.8 \pm 4$ & $0.72[9]$ & Inkjet [9] \\
Silver & $3.35 \pm 0.13[6]$ & $0.33 \times 10^{-4}[6]$ & Aerosoljet [6,7] \\
PMMA-MWCNT & $15.32[14]$ & $2.3 \times 10^{6}[14]$ & Hot pressing [14] \\
composite & $1.44[14]$ & $96.52[14]$ & n.a. \\
Epoxy-MWCNT & $2 \ldots 30[10]$ & n.a. &
\end{tabular}

It can be seen, that in case of PEDOT:PSS the electrical parameters vary significantly. Varying humidity and different finishing temperatures during the manufacturing process and characterization could be the reason. The low conductivity of the conducting polymers like PEDOT:PSS and in particular the composites can lead to resistance in the range of $M \Omega$ for the realized thin sensor layers. In case of conducting substrates, the resistance of the insulating layer will influence the measurement results. To solve the above mentioned problems of realizing printed strain sensors, we characterized the electrical properties of purchasable inks like PEDOT:PSS and silver suspensions. Both inks are available for inkjet and screen printing and have already been used for realizing strain sensors. Due to the fact, that we integrate printed sensors into conducting systems, an insulating layer with a high insulating resistance is necessary. The insulation must not be decreased by a high strain level. Thus, the electrical properties of the insulating layer according to the strain level are been investigated. Based on the results, we design a sensor.

\section{Design of the Printed Resistive Sensor}

\subsection{Requirements and Restrictions}

Two sources of requirements and restrictions respectivly can be appointed. On one hand, the printing method defines viscosity, paticle size of the ink and resolution. On the other hand, the application dictates e.g. measurement range and thus sensitivity, size and basic resistance $\mathrm{R}_{0}$ of the sensor itself. In our application, we aim on non-destructive testing of conducting lightweight structures made of steel or carbon fiber laminates. Table 2 lists the resulting basic requirements on the sensor.

Tab. 2 Basic Requirements on printed strain sensors.

\begin{tabular}{l|c|c}
\multicolumn{1}{c}{ Parameter } & Specification & Comment \\
\hline Nominal strain $S_{\mathrm{N}}$ & $\leq 500 \mu \mathrm{m} / \mathrm{m}$ & truss structure, see [2] \\
Resolution $\Delta S$ & $\leq 5 \mu \mathrm{m} / \mathrm{m}$ & max. area for 1 resistor \\
Sensor size $(w \mathrm{x} l)$ & $\leq 10 \times 10 \mathrm{~mm}^{2}$ & \\
Nonlinearity $f_{\mathrm{L}}$ & $\leq 10 \%$ & related to nominal strain $\mathrm{S}_{\mathrm{N}}$ \\
Hysteresis $f_{H}$ & $\leq 10 \%$ & piezoresistivity desired \\
Gauge factor $K$ & $\geq 2$ & $\begin{array}{c}\text { comparable to conventional } \\
\text { strain sensors }\end{array}$ \\
Sheet resistivity $R_{\square}$ & $<10 \mathrm{k} \Omega$ & $\mathrm{R}_{0}$ \\
$R_{\text {insulation }}$ & $>50 \mathrm{M} \Omega$ & dielectric layer, see [12]
\end{tabular}

\subsection{Materials and Manufacturing Process}

In our experiments we use steel (DC-04) specimens as substrates with a thickness of about $1 \mathrm{~mm}$. It is a common material for realizing chassis in automtive industry. To insulate the sensing layer, a polyester resin (Bectron DP 8401 VP, Elantas Beck) is chosen. It is a thermally cross-linking polymer, which is well known as coating material for insulating coil wires [11]. Using screen printing, an about $20 \mu \mathrm{m}$ thick layer of the resin is printed on top of the specimen and dried at $80^{\circ} \mathrm{C}$ for 30 minutes in a climate chamber. Two different conductive inks are chosen as sensing layer. The first one is a thermally cross-linking silver suspension (Nicomatic AG500, Nicomatic), which is screen printed on top of the insulation layer. It is dried at about $125^{\circ} \mathrm{C}$ for 15 minutes in a climate chamber and has a final layer thickness of about $7 \mu \mathrm{m}$. The other one is a water-based suspension of PEDOT:PSS (Clevios, H. C. Starck). It is a conductive polymer, which is a well known contacting material for organic light emitting diodes. The suspension is printed on top of the conducting layer using a Dimatix inkjet printer (DMP-2831) and dried in a climate chamber at room temperature for 24 hours. A layer thickness of about $1 \mu \mathrm{m}$ is realized. For connecting the sensing layers, insulated copper wires with a diameter of about $100 \mu \mathrm{m}$ are integrated using a conducting adhesive (Elecolit 3653, Panacol) and dried at about $100{ }^{\circ} \mathrm{C}$ for one hour in an oven. Fig. 1 shows the assembly of the test specimen. Because the manufacturing process with its subsequent drying steps influences 
the micro-structure of the printed layers, the electrical properties of the printed inks have to be investigated $[6,11]$.

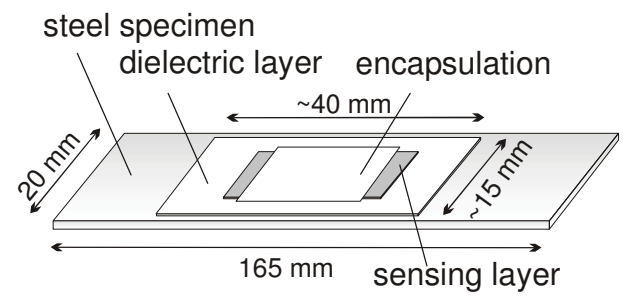

(a) Dimensions of test specimen

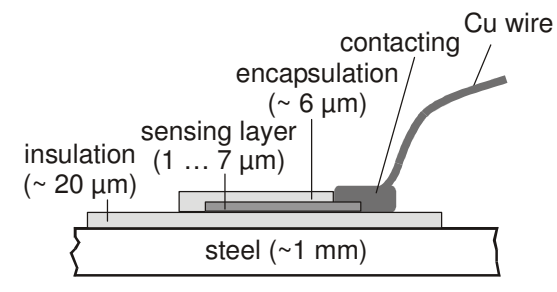

(b) Detail: packaging of a printed sensor

Fig. 1. Assembly for testing the electrical and electromechanical properties of printed resistive layers. The encapsulation prevents oxidation and water storage of the sensing layer.

\subsection{Electrical Properties of the Conducting Inks}

\subsubsection{Measurement Setup}

The resistivity $\rho$ of printed silver and PEDOT:PSS layers according to the mentioned manufacturing process has to be analyzed. Fig. 2 shows the utilized measurement setups. The resistance of about 15 sample for each ink and printing technology is measured. The manufacturing process we mentioned before is used for realizing test specimens without encapsulating. The conducting layer has a width of about $6 \mathrm{~mm}$ and a length of about $40 \mathrm{~mm}$. In case of PEDOT:PSS, the sheet resistance of the printed layers is measured in a four-wire setup shown in Fig. 2(a). Mean value and standard deviation are determined. In case of the silver layers a second setup shown in Fig. 2(b) was used. Flexible electrodes with a contacting area of $5 \times 8 \mathrm{~mm}^{2}$ (rubber coated with copper and platin layers) are clamped in a fixed distance of $32 \mathrm{~mm}$ onto the silver layer and the resistance is measured. In a preliminary test the equivalence of both measurement setups was validated. To determine the resistivity $\rho$, the layer thickness of every sample is measured using an optical profilometer (Pl $\mu$ Neox, Sensofar).

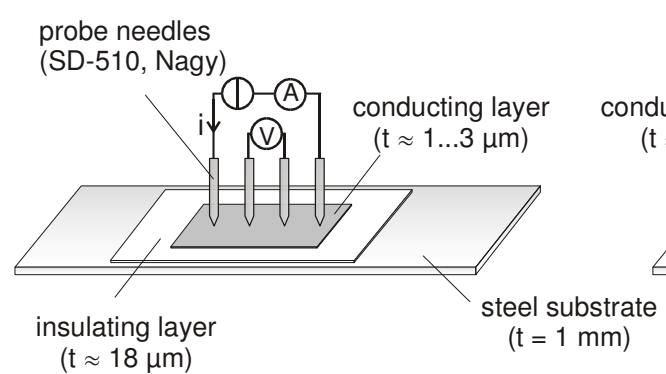

(a) Measurement of sheet resistance of PEDOT:PSS layers digital voltmeter (8840A, Fluke)

Fig. 2. Measurement setup for analyzing the resistivity of printed layers.

\subsubsection{Measurement Results}

Tab. 3 lists the measured values for resistivity and thickness. The results are compared with bulk copper.

Tab. 3 Resistivity of functional inks compared to bulk copper.

\begin{tabular}{l|c|c}
\multicolumn{1}{c|}{ Functional material } & Resistivity & Layer thickness \\
\hline PEDOT:PSS (inkjet printed) & $0.42 \Omega \mathrm{mm}$ & $50 \mathrm{~nm}+/-5 \mathrm{~nm}$ \\
PEDOT:PSS (screen printed) & $0.78 \Omega \mathrm{mm}$ & $1 \mu \mathrm{m}+/-0.5 \mu \mathrm{m}$ \\
Silver (screen printed) & $7.8 \Omega \mathrm{mm}$ & $6.8 \mu \mathrm{m}+/-1 \mu \mathrm{m}$ \\
Copper & $1.7 \cdot 10^{-5} \Omega \mathrm{mm}$ & Bulk
\end{tabular}

The resistivity of the silver suspension is about 100 times higher than that of bulk copper. In case of

PEDOT:PSS a factor of $10^{5}$ occurs. Taking these values into account, the printed sensor can be layouted.

\subsection{Topology of the Sensor}

Following the standards of conventional strain gauges, a resistance $R_{0}$ of about $120 \Omega$ up to $1 \mathrm{k} \Omega$ should be obtained. Based on the measurement results shown above, a sensor topology can be designed.

Taking achievable layer thickness $t$, printing resolution and resistivity $\rho$ into account, the sensor resistance $R_{0}$ can be determined via its resistivity $\rho$ and its geometry parameters length I, width $w$ and layer thickness. The resistivity of screen printed silver suspension is comparable to conventional sensing materials like constantan $\left(\rho \approx 4.9 \times 10^{-4} \Omega \mathrm{mm}\right)$. Printing a meander with a linewidth of $150 \mu \mathrm{m}$ and a layer 
thickness of about $7 \mu \mathrm{m}$, a basic resistance of about $120 \Omega$ can be realized. Each resistor has a length of $9.3 \mathrm{~mm}$ and a width of $4.65 \mathrm{~mm}$ (see Fig. (a)). In case of the PEDOT:PSS suspension, the resistivity is much higher. A layer thickness of $50 \mathrm{~nm}$ up to $1 \mu \mathrm{m}$ is achievable by inkjet printing several ink layers. In our case we have chosen a layer thickness of about $500 \mathrm{~nm}$. Printing a layer of about $(\mathrm{w}=600 \mu \mathrm{m}) \mathrm{x}$ $(\mathrm{t}=0.5 \mu \mathrm{m}) \times(\mathrm{l}=2 \mathrm{~mm})$, a basic resistance of about $6 \mathrm{k} \Omega$ can be realized.

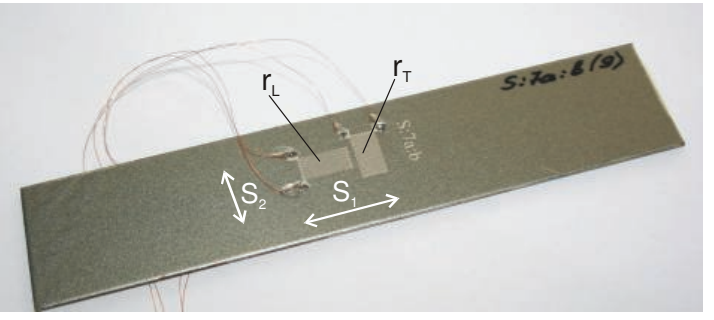

(a) Specimen for bending test with two screen printed silver sensors.

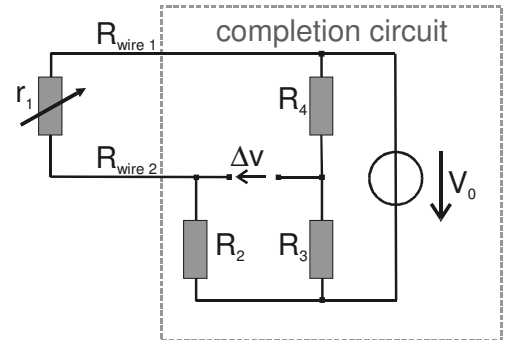

(b) $1 / 4$-bridge configuration for evaluating one strain sensing resistor.

Fig. 3. Test specimen with the chosen sensor topology. Sensor $r_{L}$ detects longitudinal strain $S_{1}$, sensor $r_{T}$ detects transversal strain $\mathrm{S}_{2}$.

A topology of two perpendicularly arranged resistors is chosen, to be able to detect a biaxial strain condition. Resistor $r_{L}$ measures longitudinal strain $S_{1}$, resistor $r_{T}$ measures transversal strain $S_{2}$ (Fig. 3(a)). The insulating of the sensing structure is done by an about $20 \mu \mathrm{m}$ thick dielectric layer. The analysis of the electrical impedance showed, that a sufficient insulation is obtained even at strain rates up to $20 \%$ [15]. Using the manufacturing process described in section 3.2, test specimens are realized.

\section{Sensor Characterization}

\subsection{Measurement Setup}

Twelve samples of the screen printed silver suspension and ten samples of the inkjet printed PEDOT:PSS suspension are investigated. Using a digital voltmeter (8840A, Fluke) the resistance of the sensors is measured. To get the characteristics of the printed sensors, the steel specimens are strained. A four-point-bending setup was designed to realize constant longitudinal strain in a defined area. The steel specimen bears on two parallel cylinders with a distance of $80 \mathrm{~mm}$. A stepper motor (Nema-8, ADrive) moves two paralell cylinders (distance $\approx 40 \mathrm{~mm}$ ) to deflect the specimen. The deflection is measured with laser triangulator (LK-G32, Keyence) with a resolution of $0.05 \mu \mathrm{m}$. Torsions of the steel specimen itself can be adjusted due to a bearing of the force transmission. Using a Labview routine, the setup is controlled via a PC. To evaluate the resistance change the use of a Wheatstone configuration of four resistors is common (Fig. 3(b)). The bridge circuit is supplied with $\mathrm{V}_{0}=5 \mathrm{~V}$. The relative change of the output voltage $\Delta v / V_{0}$ is directly propotional to the acting strain $S_{1}$. Equation 2 describes the change of bridge voltage $\Delta \mathrm{v}$ for one strain sensing resistor for longitudinal strain.

$$
\frac{\Delta v}{V_{0}} \approx \frac{1}{4} \cdot K_{1} \cdot S_{1},
$$

whereas $\mathrm{K}_{1}$ is defined as the gauge factor in longitudinal direction. A completition circuit adjusts a varying resistance of the sensor. The output voltage is amplified using a two-channel amplifier (Kistler SCP 5271), digitalized and read out using Labview. Each specimen is tested in two loading and unloading cycles up to $500 \mu \mathrm{m} / \mathrm{m}$ longitudinal strain. The transducer characteristics like gauge factor and crosssensitivity, nonlinearity and hysteresis error referring to the nominal value are calculated.

\subsection{Measurement Results}

The basic resistance $\mathrm{R}_{0}$ of the screen printed silver sensors is about ten times higher than expected. Also the resistance of the PEDOT:PSS sensors differs from the initial value (see Tab. 4). Fig. 4 shows the characteristics of a sensor made of a screen printed silver suspension. In case of the PEDOT:PSS sensors, the output voltage $\Delta \mathrm{v}$ is not proportional to the acting strain, but superimposed by a great offset drift with a drift rate of $200 \mathrm{mV} / \mathrm{h}$. A characteristic curve is not measurable.

\section{Discussion}

The basic resistance $\mathrm{R}_{0}$ of all analyzed sensors varies in a wide range. Both conducting ink and printing method can cause the variation. In the following, the two types of printed sensors will be discussed separately. In case of the silver suspension, we used screen printing. $\mathrm{R}_{0}$ is ten times higher than expected. One reason for the variations could be the manufacturing process itself. In case of screen printing, the resistance is varying according to the orientation of resistor and coating knife. If the resistor and the mov- 
ing direction of the knife are perpendicular oriented, the linewidth of the meander can be reduced. The resistance will take higher values than expected.

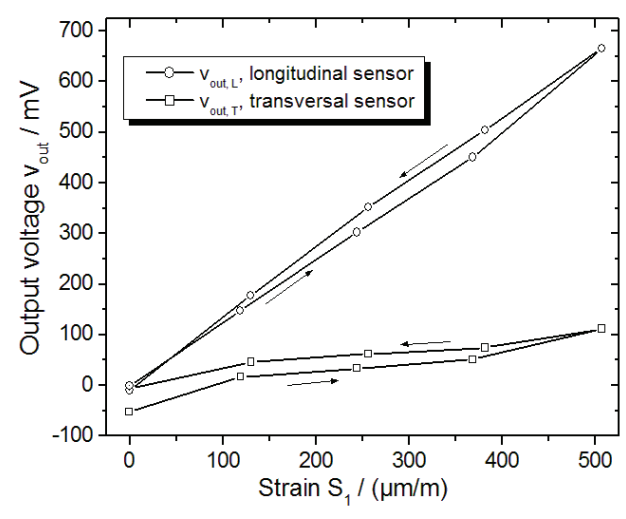

(a) Static characteristic curve of a printed sensor.

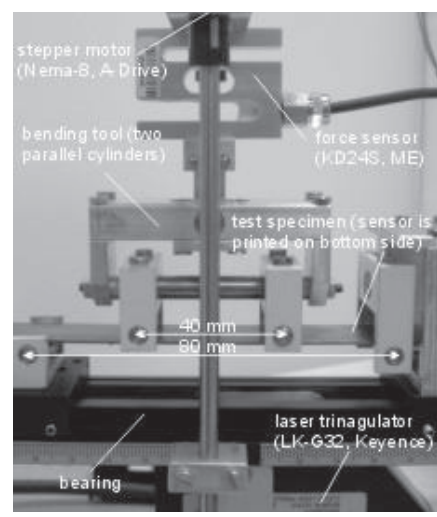

(b) Four-point-bending test setup

Fig. 4. Static characteristic curve of a printed silver sensor and measurement set-up. Strain up to 500 $\mu \mathrm{m} / \mathrm{m}$ is applied in a four-point-bending set-up. $K_{l}=1.06, Q \approx 8 \%$, nonlinearity is about $3 \%$ and hysteresis error takes a value of about $19 \%$.

This applies to resistor $r_{\mathrm{T}}$. Its linewidth is reduced and the resistance is about $200 \Omega$ higher than in case of $\mathrm{r}_{\mathrm{L}}$. Secondly, the surface texture of the insulation layer could influence the layer thickness. Due to the surface roughness, the thickness of the applied ink layer could vary. In a reproducibility test, the surface texture of insulation layer and layer thickness of silver have been analyzed at twelve samples. A locationindependent difference of up to $4 \mu \mathrm{m}$ of the layer thickness within one resistor was detected. An influence of the texture itself was not detectable. Beside the printing process, also the silver suspension could be responsable for variations. On one hand, the dispersion of the suspension or on the other hand, a differing batch, which is used for manufacturing, could lead to a varying number of silver particles per sensor. The use of a contactless printing method like inkjet or aerosoljet can reduce the variations within the topology itself. To ensure a predictable resistance, the resistivity of every batch has to be characterzied seperatly. Additionally, a (laser-)trimming of resistor, which is a common technique for adjusting the resistance of thick-film sensors, or an adjustable topology has to be taken into account. In case of the PEDOT:PSS sensor we used inkjet printing. The mean resistance of the sensors was in the expected range. On one hand, the printing method could be responsable. For realizing a layer thickness of $500 \mathrm{~nm}$, about 20 ink layers have to be printed. The thickness of every layer has a deviation of +/- 5 $\mathrm{nm}$. The final layer thickness of our sample varies in a great range of up to $70 \mathrm{~nm}$. This can lead to a differing resistance of the sensors. On the other hand, the electrical properties of the PEDOT:PSS suspension is instable [13]. The material is highly hygroscopic and even with a silicone coating the resistance is varying over time. Also, a change of temperature leads to a non-reversible resistance change [13]. Only in an inert gas atmosphere the variation of the resistance could be mimimized [13]. Hermetic ecapsulation solutions or a modification of the functional material itself have to be investigated. The sensor characteristics are measured in a four-point-bending setup (see Fig. 4(b)). For sensors made of silver suspensions we expected, that the shape changing will dominate and a gauge factor of about 2 occurs (see equation 2). But the measured gauge factor was reduced. Taking the fundamentals of resistive sensors into account, the gauge factor can be expressed by

$$
K_{1}=\underbrace{(1+2 \cdot v)}_{\text {shape-change }}+\underbrace{\frac{\Delta \rho}{\rho \cdot S_{1}}}_{\text {piezoresistivity }}=1+2 \cdot v+K_{1}^{*} \text {. }
$$

Considring the Poisson ratio $v$, the shape-changing part can be calculated. Thus, the piezoresistive part $\mathrm{K}_{1}{ }^{*}$ is the difference of the shape-changing part and the measured value. If $\mathrm{K}_{1}{ }^{*}$ takes negative values, it counteracts to the shape-changing effect. This reduces the gauge factor $K_{1}$. A second reason could be a damping beaviour of the insulating layer, so that the strain of the measuring object is insufficiently transmitted into the sensing element. Further measurements on insulating substrates will be done to eliminate the influence of the insulation layer. The cross-sensitivity of the sensors is at about $10 \%$ and thus in the range of thick-film sensors. Also nonlinearity and hysteresis error are much higher than in case of conventional strain gauges. Beside the viscoelastic behavior of the inks also varying conducting paths within the sensing layer could be responsable. Also, a differing thermal behavior of completition circuit and printed sensor can lead to higher measurement uncertainty. A completition circuit of printed sensors 
will be realized to reduce the thermal effects. Secondly, a thermal cycling of the sensor will be analyzed and its influence onto the microstructure of the sensing layer. As mentioned before in chapter 2, for the PEDOT:PSS sensors a piezoresisitve behavior could be expected. In our test setup, a characteristic curve was not detectable. First measurements show, that the resistance of the PEDOT:PSS sensors and thus the output voltage $\Delta \mathrm{v}$ is highly drifting in a range of up to $200 \mathrm{mV}$ per hour at an amplification factor of 10. This corresponds to the output voltage of a conventional strain gauge at a strain of $500 \mu \mathrm{m} / \mathrm{m}$. Besides a different thermal behavior of completition circuit and polymeric sensor, varying surrounding temperature and a leakage of the encapsulating and thus varying humidity could be responsable. Also, an influence of the conducting substrate is possible. At the moment, further investigation are done to distinguish the different influencing parameters, which disturbe the sensing effect.

Tab. 4 Transducer parameters of printed strain sensors.

\begin{tabular}{lcc} 
Parameter & Silver & PEDOT:PSS \\
\hline Basic Resistance & $\mathrm{r}_{\mathrm{L}}=0.97+1-0.02 \mathrm{k} \Omega$ & $6.4+/-02 \mathrm{k} \Omega$ \\
& $\mathrm{r}_{\mathrm{T}}=1.19+1-0.08 \mathrm{k} \Omega$ & \\
Gauge factor $\mathrm{K}_{1}$ & $1.22+1-0.06$ & n.a. \\
Piezoresistivity $\mathrm{K}_{1}{ }^{*}$ & $-0.48+/-0.08$ & n.a. \\
Cross sensitivity Q & $\geq 10 \%$ & n.a. \\
Hysteresis error $f_{\mathrm{H}}$ & $7 \ldots 15 \%$ & n.a. \\
Nonlinearity $f_{\mathrm{L}}$ & $2 \ldots 13 \%$ & n.a.
\end{tabular}

\section{Outlook}

Nonlinearity $f_{\mathrm{L}}$

Beside the analysis of the two purchasable inks, we also investigate conducting polymers like nanocomposites. Together with material scientists, we formulate siloxane-mwcnt-composites. At the moment the composites show a lack of conductivity - the basic resistance of the test specimen is in the range of $\mathrm{M} \Omega$ and not suitable for sensing applications on conducting surfaces.

\section{Acknowledgement}

The authors would like to thank the members of Deutsches Kunststoff Institut - DKI, especially Dr. T. Finnberg for the support on the topic of analyzing dielectric properties of printed sensors and valuable discussions. The work is funded by the LOEWE-AdRIA project and the state government of Hesse, Germany.

\section{References}

[1] White, N. and Turner, J., Thick-film sensors: past, present and future, Measurement Science and Technology 8 , IOP Publishing (1996).

[2] Flaschenträger, D. et al., "Implementation and characterization of the dynamic behaviour of a three dimensional truss structure for testing smart devices," International Conference on Nois and Vibration - ISMA (2010).

[3] Bai, J., Zhang, Z. Z., Calata, J. N. and Lu, G.-Q., "Low-temperature sintered nanoscale silver as a novel semiconductor device-metallized substrate interconnect material," Components and Packaging Technologies 29, 589 -593 (2006).

[4] Maiwald, M. et al., INKtelligent printed strain gauges, Sensors and Actuators A: Physical 162(2), 198-201 (2010). [5] Nocke, A., Richter, S., Wolf, M. and Gerlach, G., Polymer composite strain sensor based on dielectrophoretically aligned tellurium nanorods, Procedia Chemistry 1, Elsevier (2009).

[6] Latessa, G. et al., Piezoresistive behaviour of flexible pedot:pss based sensors, Sensors and Actuators B: Chemical 139(2), $304-309$ (2009).

[7] Pham, G. and Park, Y., Processing and modeling of conductive thermoplastics/carbon nanotube films for strain sensing, Composites: Part B 39, p. 209-216 (2008).

[8] Lang, U., Experimental methods for evaluating the mechanical properties of thin layers of intrinsically conductive polymers, PhD thesis ETH Zurich (2008).

[9] Srichan, C. et al., "Inkjet printing PEDOT: PSS using desktop inkjet printer," in ECTI-CON 2009. 6 th International Conference on], 1, 465-468, IEEE (2009).

[10] Kus, M. and Okur, S., "Electrical characterization of pedot: Pss beyond humidity saturation," Sensors and Actuators B: Chemical 143(1), 177-181 (2009).

[11] Salun, L. et al., "Mechanical and electrical stability of printed layers against mechanical deformation," Large-area, Organic and Printed Electronics Convention (LOPE-C) (2010).

[12] Hoffmann, K., Eine Einführung in die Technik des Messens mit Dehnungsmessstreifen, HBM Verlag (1985).

[13] Nardes, A. M., On the conductivity of PEDOT:PSS thin films, PhD thesis, Technische Universiteit Eindhoven (2007).

[14] Hu, N. and Karube, Y., A strain sensor from Polymer/Carbon Nanotube Nanocomposite, Carbon 48 S, p. $680-$ 687 (2010).

[15] Rausch, J. et al., Printed resistive strain sensors for monitoring of light-weight structures, SPIE Conference on SPIE Smart Structures/NDE, San Diego (2011) 